\title{
Umfassende Therapie psychotischer Störungen (Teil I)
}

\footnotetext{
$\mathrm{D}$
} ie Behandlung von Menschen mit psychotischen Störungen hat sich in den letzten Jahren deutlich gewandelt. Dies betrifft nicht nur die Inhalte der Therapie, sondern vor allem auch deren notwendige institutionelle Rahmenbedingungen. Studien zur Früherkennung haben gezeigt, dass die Mehrzahl der Menschen mit einer späteren psychotischen Erstmanifestation zuvor schon lange unter zunächst häufig unspezifischen prodromalen und nachfolgend unter unerkannten psychotischen Symptomen leiden. Die Früherkennung mit einer möglichst früh beginnenden Behandlung bietet hier die Möglichkeit, die psychosozialen Folgeschäden dieser Phase der unbehandelten Erkrankung zu verhindern bzw. zu minimieren. Zu welchem Zeitpunkt neben psychosozialen Interventionen eine medikamentöse Frühbehandlung begonnen werden soll, bleibt allerdings weiterhin umstritten und muss daher unter Berücksichtigung aller Risikofaktoren individuell entschieden werden.

Darüber hinaus wurde durch Studien belegt, dass viele Patienten schon im jugendlichen oder jungerwachsenen Alter erkranken. Um deren besonderen Bedürfnissen besser gerecht zu werden, wurden in vielen Ländern spezielle Institutionen zur altersübergreifenden Behandlung in Zusammenarbeit zwischen Kinder- und Jugendpsychiatrie und Erwachsenenpsychiatrie geschaffen. Ein wichtiges Ziel solch spezialisierter Zentren ist zudem die Verbesserung der Qualität der Behandlung. Wie in vielfachen Studien gezeigt, ist eine integrierte, settingübergreifende, multimodale Behandlung einer Standardbehandlung deutlich überlegen. Integriert bedeutet dabei, dass jeder Patient inklusive seiner Angehörigen ein individuelles, bedürfnisorientiertes und langfristiges Therapieangebot bekommt.

Im Sinne einer integrativen Therapie müssen eine Reihe von Behandlungsoptionen angeboten werden. Zum Behandlungsstandard gehören u.a. eine individuelle, settingübergreifende, langfristige und aufsuchende Bezugstherapie, qualifizierte Pharmakotherapie, Psychoedukation, Familienintervention, störungsspezifische psychotherapeutische Einzel- oder Gruppeninterventionen oder Angehörigengruppen. Da bei vielen Patienten kognitive Dysfunktionen, komorbide psychische Störungen und soziale Defizite bestehen, sind zum Beispiel auch kognitives Training, Suchttherapie oder Sozia- les Kompetenztraining sinnvoll. Wesentliche Probleme der Behandlung sind die medikamentöse Non-Compliance und der Behandlungsabbruch. Hier haben sich präventive Ansätze und das so genannte Assertive Community Treatment (ACT) als sinnvoll erwiesen. Da viele Patienten schon bei Erstbehandlung ohne Arbeit oder Schulabschluss sind und das Erreichen solcher Ziele für eine gute Lebensqualität wichtig ist, sind auch dementsprechende qualifizierte (Früh)Reintegrationsprogramme notwendig.

Teil I dieses Heftes fokussiert die Langzeittherapie Behandlungssetting, medikamentöse Therapie, Prävention und Behandlung von Non-Compliance sowie psychosoziale Interventionen - und versucht eine umfassende Therapie für Menschen mit einer psychotischen Störung darzustellen.

Teil II im Heft 07/06 beschreibt die Frühbehandlung, Remission und Lebensqualität als Erfolgsparameter sowie die Behandlung von komorbiden Störungen.

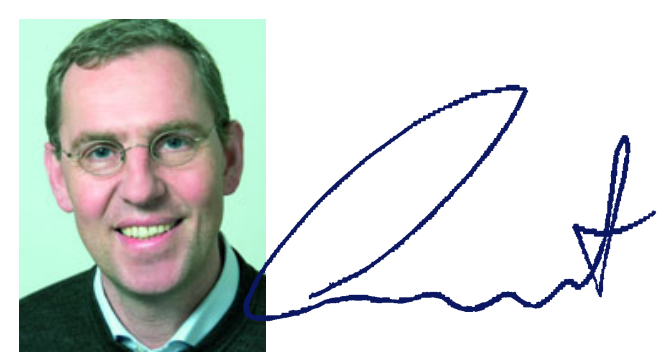

PD Dr. Martin Lambert, Hamburg

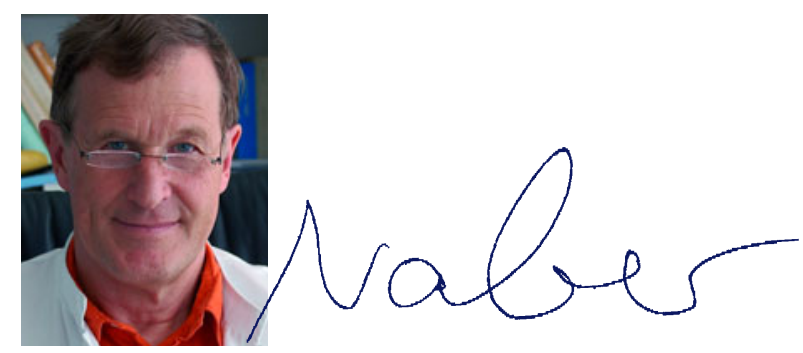

Prof. Dr. Dieter Naber,

Hamburg 